\title{
PLANES INDIVIDUALES DE APOYO: MEJORA DE LOS RESULTADOS PERSONALES
}

\section{Individual Support Plans: enhancing personal outcomes}

Jos H. M. van LoON, PHD.

Fundación Arduin y Departamento de Educación Especial de la Universidad de Gante. Koninginnelaan 50.4335 BC Middelburg. The Netherlands jloon@arduin.nl

Recepción: 27 de noviembre de 2014

Fecha de aceptación definitiva: 1 de diciembre de 2014

Biblid. [0210-1696 (2015) vol. 46 (1), n. ${ }^{\circ} 253$, enero-marzo; 25-40]

Resumen: La mejora de la calidad de vida de los usuarios es la principal tarea de las organizaciones que prestan servicios de apoyo a las personas con discapacidad intelectual. Los planes individuales de apoyo (PIA) deben diseñarse teniendo en cuenta este objetivo. Por tanto, las cuestiones más importantes a las que debe prestarse atención cuando se redacta un PIA son: 1) ¿Qué quiere el usuario? y 2) ¿Qué apoyos necesita? Obviamente, lo que se busca es que el usuario goce de una buena calidad de vida.

Además, en este trabajo hacemos hincapié en la importancia de las prácticas basadas en la evidencia; y, sobre la base de estos dos puntos de partida, proponemos las siguientes directrices para la elaboración del PIA. En el PIA se debe:

- Analizar los objetivos y perspectivas del usuario (¿qué quiere conseguir en la vida?).

- Analizar qué apoyos el usuario quiere y necesita (¿qué apoyos son importantes desde su punto de vista y cuáles realmente necesita?).

- Formular estrategias de apoyo en función de los objetivos personales, los deseos y las necesidades del usuario.

- Hacer un seguimiento de los procesos de apoyo en permanente diálogo y colaboración con el usuario.

- Medir los resultados personales que se han conseguido.

- Disponer de un sistema permanente para llevar a cabo los ajustes y adaptaciones que sean necesarios. 
- Recurrir a las prácticas basadas en la evidencia.

- Ser claro y comprensible para el usuario.

Con estas directrices hemos desarrollado un PIA en Internet, en el cual las ocho dimensiones de calidad de vida conforman el marco teórico a partir del cual se elaboran las estrategias de apoyo. El diálogo con el usuario se integra en el sistema de apoyo y los instrumentos basados en la evidencia se utilizan para medir las necesidades de apoyo y los resultados personales obtenidos.

Palabras Clave: prácticas basadas en la evidencia, calidad de vida; planes individuales de apoyo; resultados personales; equipos de alto rendimiento.

ABSTRACT: For organizations providing supports to persons with intellectual disability it is their main task to enhance the quality of life of their clients. Individual Support Plans should be designed with this goal in mind. Consequently, important questions in writing an ISP are: 1) What does the person want?, and 2) Which support does the person need? The outcome should obviously be a good quality of life.

Furthermore we emphasize in our work the importance of evidence-based practices. Based on these starting points we propose the following guideliness for an ISP. An ISP should:

- Explore goals and personal perspectives: what does a person want in his life.

- Explore what support a person needs and wants: which supports are important for and which are important to the person.

- Formulate support strategies in answer to the personal goals, wants and needs.

- Monitor in dialogue with the person the process of support.

- Measure personal outcomes.

- Comprise an ongoing system of finetuning and adjusting.

- Use evidence based practices in doing so.

- Be transparant and comprehensible for the person.

Following these guideliness we developed an internet based ISP in which the eight QOL dimensions provide the framework for developing support strategies, the dialogue with the client is build into the system of supports, and evidence based instruments are used to measure support needs and personal outcomes.

KEY WORDs: evidence-based practices; quality of life; Individual Support Plans; personal outcomes.

\section{Introducción}

P ARA LAS ORGANIZACIONES, proporcionar apoyos a las personas con discapacidad es su tarea principal con la vista puesta en mejorar la calidad de vida de los usuarios. Por otro lado, uno de los retos actuales a los que deben hacer frente estas organizaciones, y a la vez un aspecto al que cada vez se da más importancia, es la necesidad de utilizar prácticas basadas en la evidencia. Las prácticas basadas en la evidencia (PBE) se definen como las prácticas que se fundamentan en la mejor evidencia actual obtenida a partir de fuentes creíbles que utilicen métodos válidos y fiables y un método o fundamento teórico claramente articulado y empíricamente refrendado (Schalock, Verdugo y Gómez, 2011). Los resultados basados en la evidencia (RBE) 
son ahora un componente muy importante de las prácticas basadas en la evidencia (Van Loon et al., 2012). Los resultados basados en la evidencia se definen como las medidas obtenidas a partir de la evaluación de los indicadores centrados en los dominios de calidad de vida, que a) se basan en un modelo conceptual y de medición de la calidad de vida (CV) validado transculturalmente (Schalock, Verdugo, Jenaro, Wang, Wehmeyer, Xu et al., 2005; Wang, Schalock, Verdugo y Jenaro, 2010); b) son útiles porque pueden utilizarse para múltiples propósitos (Schalock, Verdugo, Bonham, Fantova y Van Loon, 2008; Van Loon, Claes, Van Hove y Schalock, 2010); y c) son robustos en lo que respecta a la validez y la fiabilidad de la estrategia de evaluación utilizada (Claes, Van Hove, Van Loon, Vandevelde y Schalock, 2009).

Las prácticas basadas en la evidencia y los resultados basados en la evidencia están relacionados respectivamente con los componentes proceso y salidas/resultados de un modelo lógico de programa.

Esto quiere decir que los apoyos proporcionados por un proveedor de servicios deben estar basados en la evidencia y, a la vez, dar lugar a buenos resultados basados en la evidencia: buenos resultados personales relacionados con la calidad de vida.

Este artículo se centra en la aplicación de los resultados basados en la evidencia a la evaluación y el uso de los resultados personales relacionados con la calidad de vida y su utilización en la planificación de los apoyos individuales. En él, se explicará el Sistema de los Apoyos centrado en la persona resultante.

\section{Contexto}

Arduin es una organización holandesa que proporciona servicios para las actividades de la vida diaria. Trabaja para 740 personas con discapacidad intelectual, 535 de las cuales reciben servicios/apoyos durante las 24 horas del día. Hace unos quince años se produjo un cambio en Arduin: se pasó de los servicios centrados en las instalaciones de la organización a un procedimiento de apoyos individualizados centrados en el entorno inmediato del usuario dentro de un marco basado en la calidad de vida (Van Loon y Van Hove, 2001). Arduin ha desarrollado un marco organizativo que se centra en mejorar los resultados basados en la evidencia en el nivel de la persona.

Los supuestos fundamentales son básicos para el compromiso que una organización tiene con la calidad de vida y la provisión de apoyos individualizados. Estos supuestos son que: a) la calidad de vida de las personas con discapacidad se compone de los mismos factores (es decir, los dominios clave) y relaciones que son importantes para todas las personas; b) la calidad de vida mejora cuando se da a las personas más autonomía y capacidad de acción para participar en las decisiones que afectan a su vida; c) la calidad de vida mejora cuando se acepta y se integra plenamente a las personas en el lugar en el que viven; y d) la calidad de vida mejora gracias a la prestación de apoyos individualizados (Brown y Brown, 2003; O’Brien y O’Brien, 1989; Schalock, Brown, Cummins, Mattikka, Felce y Brown, 2000; Schalock y Verdugo, 2012 ; Vreeke, Janssen, Resnick y Stolk, 1998).

Arduin ha incorporado plenamente la mejora de la calidad de vida en las políticas y prácticas de la organización, y la implicación de los usuarios en funciones significativas para ellos se ha convertido en una estrategia esencial de la organización. La cuestión

(c) Ediciones Universidad de Salamanca

Siglo Cero, vol. 46 (1), n. 253, 2015, enero-marzo, pp. 25-40 
clave es el grado en el cual los usuarios se implican en el desarrollo y puesta en práctica de los planes individuales de apoyo (PIA). Arduin ha desarrollado un Sistema de Apoyos Individualizados que tiene las siguientes características: está centrado en la persona (es decir, se basa en los intereses, preferencias, necesidades y red de apoyos naturales de la persona); es receptivo (es decir, se basa en el diálogo entre la persona y los individuos implicados en el plan de apoyos); es flexible a lo largo de las diferentes fases de la vida; es proactivo (es decir, iguala las oportunidades con las de los ciudadanos sin discapacidad, da autonomía y capacidad de decisión a las personas, genera una inclusión social eficaz y aumenta la participación en el entorno social inmediato y en la sociedad), y se basa en datos (se basa en el patrón y la intensidad de las necesidades evaluadas de apoyos y es evaluado en términos de los resultados personales obtenidos relacionados con la calidad de vida).

Los resultados de un plan individual de apoyo para una persona deben consistir en una mejora de la calidad de vida. Para determinar si se han conseguido estos resultados, es necesario llevar a cabo una evaluación válida y fiable de los dominios relacionados con la calidad de vida. La Personal Outcomes Scale (POS [Escala de Resultados Personales]; Van Loon, Van Hove, Schalock y Claes, 2008), que se basa también en el modelo conceptual de CV y en el marco de medición de Schalock y Verdugo (2002), se elaboró para este propósito.

En el sistema de apoyos centrado en la persona existe un alineamiento entre los deseos y la metas de la persona y sus necesidades de apoyos tal como se miden mediante la Supports Intensity Scale [Escala de Intensidad de los Soportes] (Thompson, Bryant, Campbell, Craig, Hughes, Rotholz et al., 2004), el Plan Individual de Apoyo, PIA, y su calidad de vida medida mediante la POS.

Este alineamiento entre los deseos y las metas, las necesidades evaluadas de apoyos, los componentes del Plan Individual de Apoyo y los resultados en calidad de vida constituye una excelente oportunidad para mejorar las decisiones clínicas con respecto a cómo apoyar metódicamente a las personas para mejorar su calidad de vida. En Arduin, este sistema de apoyos se comunica electrónicamente mediante aplicaciones web (Van Loon, Van Hove y Schalock, 2009).

Un procedimiento basado en las páginas web para la elaboración del plan individual de apoyos basado en la valoración estandarizada de las necesidades de apoyos, las metas personales y los apoyos individualizados y para la medición de los resultados en calidad de vida

Al elaborar el Plan Individual de Apoyo (PIA), las dos preguntas más importantes y, a la vez, más sencillas, son: 1) ¿Qué quiere la persona? y 2) ¿Qué apoyos necesita? Por tanto, la tercera pregunta es: 3 ) ¿Cuál es el propósito de los apoyos? ¿Cuál debería ser el resultado obtenido con los apoyos que se proporcionan a la persona? Y también aquí la respuesta es sencilla: el resultado debería ser, obviamente, una buena calidad de vida.

Si estas son las preguntas de partida, se pueden proponer las siguientes directrices para el desarrollo del PIA. Un PIA debe: 
- Analizar las metas y las perspectivas personales: ¿qué quiere la persona en la vida?

- Analizar qué apoyo necesita y quiere la persona: ¿qué apoyos son objetivamente importantes para la persona y cuáles son importantes desde su perspectiva?

- Formular las estrategias de los apoyos en respuesta a las metas, los deseos y las necesidades personales.

- Supervisar en el diálogo con la persona el proceso de apoyo.

- Medir los resultados personales obtenidos.

- Disponer de un sistema permanente de adaptación y ajuste.

- Durante todo el proceso, utilizar las prácticas basadas en la evidencia.

- Ser transparente y comprensible para la persona.

Siguiendo estas directrices, hemos desarrollado una aplicación de Internet para el PIA, en la que las ocho dimensiones de CV proporcionan el marco para desarrollar las estrategias de los apoyos, el diálogo con el usuario se incorpora al sistema de los apoyos y se utilizan instrumentos basados en la evidencia para medir las necesidades de apoyo y los resultados personales obtenidos.

En la Figura 1 se presenta el esquema de este PIA.

\section{Figura 1. Esquema de un PIA}

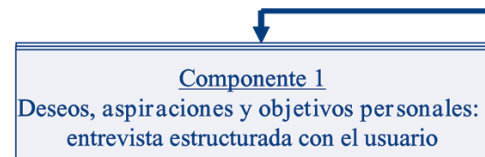

Componente 2

2.a. Determinación de las necesidades de apoyo: para la persona y desde su perspectiva Ejemplo: entrevista con la escala de intensidad de apoyos 2.b. Si es necesario: evaluación/diagnóstico adicionales

Componente 3: Desarrollo de un Plan Individual de Apoyo

a. El usuario (con su asistente personal) sintetiza los deseos y objetivos con las necesidades de apoyos y plasma su idea del plan individual de apoyos: ¿cómo quiero recibir los apoyos?

b. Esta idea se trata con los profesionales o trabajadores que prestan los apoyos (y el psicólogo).

c. Conjuntamente acuerdan un PIA sobre el apoyo que desea la persona para que pueda participar completamente en la comunidad.

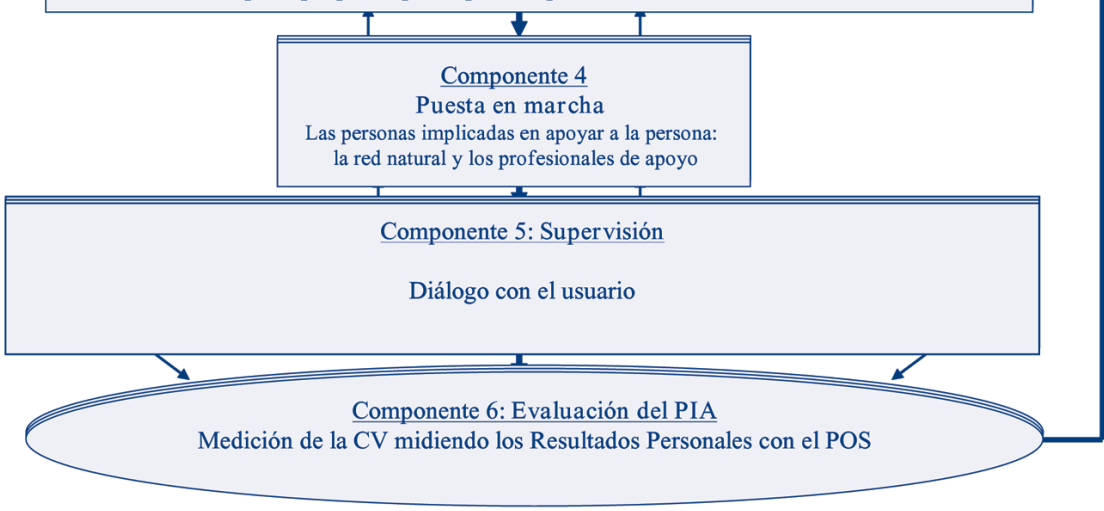

(C) Ediciones Universidad de Salamanca

Siglo Cero, vol. 46 (1), n. ${ }^{\circ} 253,2015$, enero-marzo, pp. 25-40 


\section{El diálogo como proceso central}

Como ya hemos dicho, la cuestión clave es el grado de implicación del usuario en el desarrollo y puesta en práctica de su propio Plan Individual de Apoyos (PIA). Por tanto, el elemento central en una metodología de apoyos es el diálogo con la persona. Este diálogo debe mantenerse a lo largo de todo el proceso de desarrollo del PIA y durante la prestación de los apoyos como tales. De hecho, dado que los apoyos pueden ajustarse en cualquier momento, como consecuencia de este diálogo, el proceso de desarrollo del PIA es un proceso de carácter continuo y permanente. El asistente personal es el profesional responsable del diálogo con la persona sobre el PIA. Cada usuario tiene su propio asistente al que puede llamar para que le ayude en el diálogo con la organización, así como para formular deseos y peticiones de apoyo dirigidas a esta. El asistente personal mantiene contacto de forma regular con el usuario para hablar de sus deseos (sobre la vida diaria, el trabajo y el tiempo libre) y sobre la forma de plantearlos y darles satisfacción. Los resultados de este diálogo se pueden anotar en el Plan Personal del Usuario. El asistente mantiene la comunicación entre los diferentes miembros del personal de la organización, el usuario y la familia o tutor legal. Controla que los servicios y la atención acordados son conformes con los deseos del usuario. En consecuencia, es crucial que el asistente personal no sea uno de los cuidadores directos del usuario, sino, en la mayoría de los casos, un profesional de la organización implicado directamente en la prestación de los apoyos que trabaja con otros usuarios. El asistente personal, cuando se le requiere, es el intérprete/traductor de los deseos del usuario, pero siempre explícitamente desde su rol de asistente. La intensidad y la frecuencia de este apoyo dependen totalmente de la demanda o petición del usuario (como es lógico, siempre dentro de los márgenes económicos de la organización).

En el esquema del PIA, el diálogo continuo con la persona y su asistente personal tiene un lugar explícito en el Componente 1, en el cual el centro de atención está en las metas de las persona como elemento inicial del PIA, en el Componente 3, en el que el centro de atención reside en cómo la persona quiere que se le ayude, y en el Componente 5, en el cual el centro de atención es si la persona consigue la ayuda que necesita y que le servirá para aproximarse a la consecución de las metas que tiene en la vida.

En el Componente 2, en el cual el centro de la atención está en qué apoyos necesita la persona, y en el Componente 6, cuyo centro de atención son los resultados personales, se administran la Escala de Intensidad de Apoyos y la Escala de Resultados Personales, también en una conversación con la persona, por parte de entrevistadores independientes y entrenados para esta tarea. Sin embargo, estas entrevistas se realizan una vez en un ciclo de tres años (SIS) o en un ciclo de año y medio (POS).

\section{Componente 1: las metas de la persona}

El punto de partida de un PIA es el diálogo con la persona sobre sus deseos, metas, sueños y aspiraciones personales en la vida. Es importante que nos formemos 
una imagen de lo que la persona quiere en la vida: cómo ve su futuro y cómo sueña con él. No se trata de una simple conversación, sino de uno de los temas centrales de un diálogo permanente. En la aplicación del PIA, hay una subaplicación en la que el asistente personal anota los deseos, metas y sueños que la persona va mencionando en los encuentros con él. Esta subaplicación utiliza un formato de los ochos dominios de CV. Para ayudar al asistente personal a saber dónde debe anotar qué deseos y metas, hay para cada dominio una lista de ítems de la SIS que se corresponde con el dominio de CV. Estas listas se basan en el esquema que figura en la Tabla 1.

\begin{tabular}{|l|l|}
\hline \multicolumn{2}{|c|}{ TABLA 1. Dominios de calidad de vida y los correspondientes ítems del SIS } \\
\hline \multicolumn{1}{|c|}{ Dominio de calidad de vida } & \multicolumn{1}{c|}{ Ítem del SIS } \\
\hline Desarrollo personal & $\begin{array}{l}\text { A1 - A8 (Vida en casa) } \\
\text { C1 - C6 (Aprendizaje durante toda la vida) } \\
\text { Protección y defensa legal 2 }\end{array}$ \\
\hline Autodeterminación & $\begin{array}{l}\text { C8 (Aprendizaje durante toda la vida) } \\
\text { Protección y defensa legal 1, 5 y 7 }\end{array}$ \\
\hline Relaciones interpersonales & $\begin{array}{l}\text { B4, B7 (Vida fuera de casa) } \\
\text { D3, D4 (Empleo) } \\
\text { F1 - F7 (Actividades sociales) }\end{array}$ \\
\hline Inclusión social & $\begin{array}{l}\text { B1, 2, 3, 5, 6, 8 (Vida fuera de casa) } \\
\text { F8 (Actividades sociales) }\end{array}$ \\
\hline Derechos & Protección y defensa legal 3, 4, 6, 8 \\
\hline Bienestar emocional & $\begin{array}{l}\text { C9 (Aprendizaje durante toda la vida) } \\
\text { E8 Necesidades de apoyos conductuales } \\
\text { excepcionales }\end{array}$ \\
\hline Bienestar físico & C7 E1 - E7 (Salud y seguridad) \\
Necesidades de apoyos médicos excepcionales & D1, 2, 5, 6, 7, 8 (Empleo) \\
\hline Bienestar material & \\
\hline
\end{tabular}

Además, se pide a la persona que piense sobre la importancia de cada una de las metas para poder priorizarlas. El asistente personal formula la meta/deseo dentro con un número limitado de palabras y, si es necesario, puede añadir alguna aclaración, tal como se muestra a continuación: 


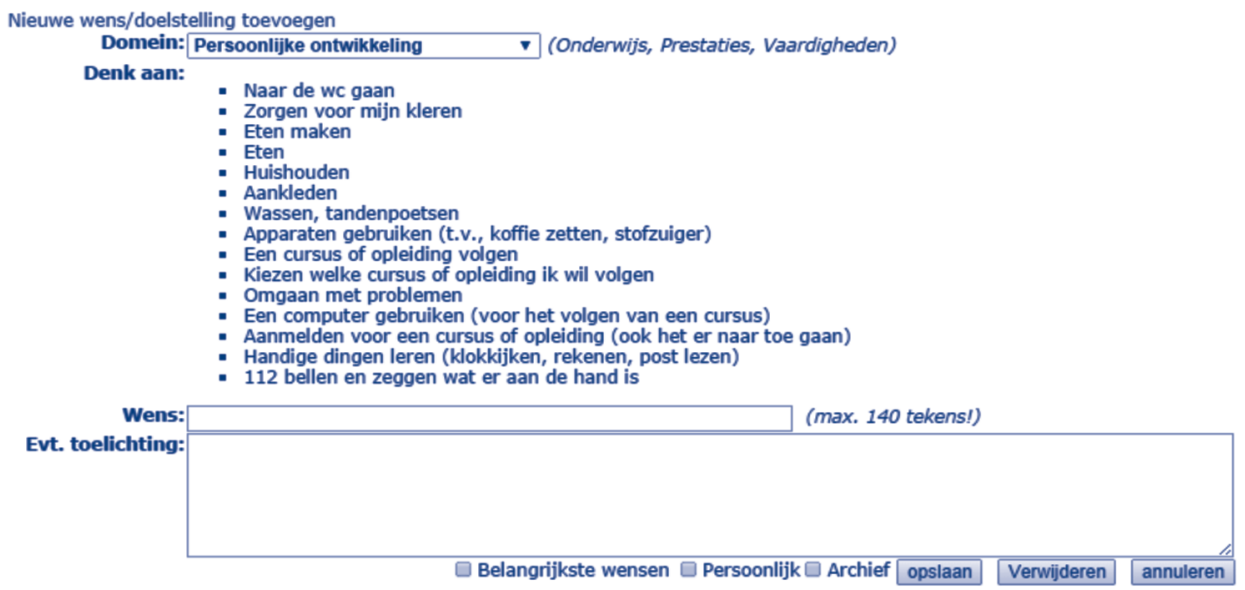

Componente 2: los apoyos que necesita la persona

Los apoyos que necesita la persona se miden mediante la Escala de Intensidad de Apoyos (SIS; Thompson et al., 2004). La SIS mide el tipo, frecuencia e intensidad de los apoyos que la persona necesita. Se compone de tres apartados:

- Apartado 1 (Support Needs Scale [Escala de Apoyos Necesarios]). Está formada por 49 actividades de la vida agrupadas en seis subescalas: 1) Vida en casa, 2) Vida fuera de casa, 3) Aprendizaje durante toda la vida, 4) Empleo, 5) Salud y seguridad y 6) Actividades sociales.

- Apartado 2, formado por ítems relacionados con las Actividades de protección y defensa legal.

- Apartado 3, Necesidades de apoyos médicos y conductuales excepcionales, incluye 15 dolencias médicas y 13 problemas de comportamiento que requieren un incremento del nivel de los apoyos.

Para cada ítem de los apartados 1 y 2, se registran el tipo de apoyo, la frecuencia y el tiempo diario de apoyo. En el caso de los ítems del apartado 3, se registra la intensidad de los apoyos.

La entrevista con la SIS se lleva a cabo explícitamente con la persona y su red social, fundamentalmente con los padres u otros miembros de la familia.

La entrevista con la SIS la realiza el MEE a cada uno de los nuevos usuarios (el MEE es una organización holandesa independiente que se dedica al apoyo social a las personas con discapacidad). Luego, la entrevista de la SIS se hace cada tres años.

Arduin ha desarrollado, con licencia de la AAIDD, una versión electrónica de la traducción al holandés de la SIS.

Cuando hay un esquema basado en la SIS de las necesidades de apoyos, el asistente personal se reúne: 1) con la persona para hablar sobre el punto de vista de esta sobre sus necesidades más importantes de apoyos (importantes según la persona) y 2) con los 
profesionales directamente implicados en la prestación de los apoyos para hablar sobre cuáles son, en opinión de estos profesionales, las necesidades más importantes de apoyo (importantes para la persona). Esta priorización se lleva a cabo en la aplicación del PIA.

\section{Componente 3: desarrollo del Plan Individual de Apoyo}

El PIA es redactado por el asistente personal en un formato de ocho dominios de CV. Junto con el usuario, se sintetizan los deseos y metas con las necesidades de apoyos y se obtiene, como resultado, una idea de cómo puede ser el plan individual: ¿Cómo se puede ayudar a una persona que quiere que se la ayude? Esta idea se discute con los trabajadores/profesionales de los apoyos y con el psicólogo y, como equipo de apoyo, deciden el PIA qué debe elaborarse con los apoyos que hay que dar a la persona para que puede participar plenamente en su entorno social.

El componente esencial en el desarrollo de estas instrucciones de apoyos es la capacidad del equipo de apoyo de resolver problemas o, mejor deberíamos decir, la capacidad y creatividad de encontrar soluciones. Para ayudar al asistente personal a redactar estas instrucciones, hay en la aplicación un compendio de ejemplos de estrategias de apoyos organizado por dominio del CV de Schalock (2014). Este compendio se muestra en la Tabla 2.

\begin{tabular}{|c|c|c|}
\hline $\begin{array}{c}\text { Dominio de calidad } \\
\text { de vida }\end{array}$ & Ejemplos de estrategias de apoyos & Efectos previstos \\
\hline Desarrollo personal & $\begin{array}{l}\text { Facilitar el establecimiento de metas } \\
\text { personales } \\
\text { Poner en práctica programas de auto- } \\
\text { gestión, autoevaluación y autoinstruc- } \\
\text { ciones } \\
\text { Construir a partir de los puntos fuertes } \\
\text { de la persona (p. ej., competencias prác- } \\
\text { ticas, habilidades sociales, experiencias } \\
\text { que han tenido éxito, capacidad de } \\
\text { compartir conocimientos) } \\
\text { Maximizar los incentivos (p. ej., recom- } \\
\text { pensa oportunidades de tener éxito). } \\
\text { Poner en práctica programas de desa- } \\
\text { rrollo de habilidades } \\
\text { Proporcionar tecnología de ayuda (p. } \\
\text { ej., dispositivos de comunicación, orde- } \\
\text { nadores, ayudas para la memoria, dis- } \\
\text { pensadores de medicación, monitores } \\
\text { de alerta médica) } \\
\text { Modificar o acomodar el entorno (casa, } \\
\text { trabajo, lugares de ocio) }\end{array}$ & $\begin{array}{l}\text {-Facilitar la motivación y el locus inter- } \\
\text { no de control } \\
\text {-Mejorar el rendimiento e incrementar } \\
\text { la sensación de autoeficacia (creencia de } \\
\text { que podemos hacer lo que nos propo- } \\
\text { nemos) } \\
\text {-Aumentar la motivación personal y la } \\
\text { capacidad de establecer metas y objetivos } \\
\text {-Facilitar el aprendizaje, la independen- } \\
\text { cia, las interacciones y la comunicación } \\
\text {-Aumentar el acceso, el uso y la inde- } \\
\text { pendencia }\end{array}$ \\
\hline
\end{tabular}




\begin{tabular}{|c|c|c|}
\hline $\begin{array}{c}\text { Dominio de calidad } \\
\text { de vida }\end{array}$ & Ejemplos de estrategias de apoyos & Efectos previstos \\
\hline Autodeterminación & $\begin{array}{l}\text { Permitir/facilitar el proceso de elección } \\
\text { y de toma de decisiones } \\
\text { Enseñar técnicas de autorregulación } \\
\text { Utilizar tecnología inteligente }\end{array}$ & $\begin{array}{l}\text {-Facilitar el locus de control interno, la } \\
\text { autoestima y la sensación de autonomía } \\
\text {-Mejorar el control personal }\end{array}$ \\
\hline $\begin{array}{l}\text { Relaciones } \\
\text { interpersonales }\end{array}$ & $\begin{array}{l}\text { Utilizar dispositivos de medios de co- } \\
\text { municación/sociales } \\
\text { Implicarse en programas de entrena- } \\
\text { miento en habilidades sociales } \\
\text { Implicarse en grupos de iguales (p. ej., } \\
\text { PALS, Best Buddies) } \\
\text { Maximizar la implicación de la familia } \\
\text { Enfatizar los puntos fuertes personales } \\
\text { (p. ej., actitudes, habilidades, capacidad } \\
\text { de compartir conocimientos) }\end{array}$ & $\begin{array}{l}\text {-Aumentar la implicación social } \\
\text {-Aumentar la participación en las redes } \\
\text { sociales } \\
\text {-Aumentar la contribución a la socie- } \\
\text { dad percibida por la persona }\end{array}$ \\
\hline Inclusión social & $\begin{array}{l}\text { Acceder a/interactuar con los apoyos } \\
\text { naturales } \\
\text { Utilizar las redes sociales y otros me- } \\
\text { dios sociales } \\
\text { Facilitar el transporte } \\
\text { Utilizar prótesis (sensoriales o motoras) }\end{array}$ & $\begin{array}{l}\text {-Aumentar el acceso al entorno social } \\
\text { próximo, el acceso, la participación y la } \\
\text { implicación }\end{array}$ \\
\hline Derechos & $\begin{array}{l}\text { Defender la plena ciudadanía, adquirir } \\
\text { derechos y obligaciones } \\
\text { Implicarse en la propia defensa legal } \\
\text { Tratar con respeto (p. ej., respeto a la } \\
\text { vida privada, reconocimiento de los de- } \\
\text { más y de su dignidad) }\end{array}$ & $\begin{array}{l}\text {-Garantizar la igualdad, la inclusión y } \\
\text { los derechos legales } \\
\text {-Maximizar la capacidad de decisión y } \\
\text { la inclusión } \\
\text {-Respeto por los derechos humanos }\end{array}$ \\
\hline Bienestar emocional & $\begin{array}{l}\text { Proporcionar entornos seguros y pre- } \\
\text { decibles } \\
\text { Maximizar los incentivos (p. ej., recom- } \\
\text { pensas, reconocimiento, oportunidades } \\
\text { de tener éxito, agradecimientos) } \\
\text { Utilizar apoyos conductuales positivos } \\
\text { Acceso a los servicios profesionales }\end{array}$ & $\begin{array}{l}\text {-Reducir los miedos y la ansiedad } \\
\text {-Aumentar la motivación y el grado de } \\
\text { satisfacción } \\
\text {-Disminuir los comportamientos pro- } \\
\text { blemáticos e incrementar las interaccio- } \\
\text { nes positivas } \\
\text {-Mejorar la salud mental/conductual }\end{array}$ \\
\hline Bienestar físico & 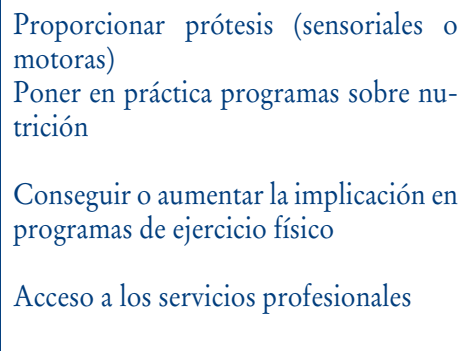 & $\begin{array}{l}\text {-Aumentar el procesamiento sensorial } \\
\text { y la movilidad física } \\
\text {-Mantener el control del peso corporal } \\
\text { y animar a seguir una dieta completa y } \\
\text { equilibrada } \\
\text {-Mejorar el funcionamiento humano y } \\
\text { reducir los efectos negativos de la obe- } \\
\text { sidad y/o la inactividad } \\
\text {-Mantener o mejorar la forma física y el } \\
\text { estado fisiológico }\end{array}$ \\
\hline
\end{tabular}




\begin{tabular}{|l|l|l|}
\hline $\begin{array}{c}\text { Dominio de calidad } \\
\text { de vida }\end{array}$ & \multicolumn{1}{|c|}{ Ejemplos de estrategias de apoyos } & \multicolumn{1}{c|}{ Efectos previstos } \\
\hline Bienestar material & $\begin{array}{l}\text { Participar en programas de empleo pro- } \\
\text { tegido } \\
\text { Proporcionar empleo remunerado en } \\
\text { talleres protegidos y supervisados } \\
\text { Red de empleadores genéricos } \\
\text { Participación en programas de forma- } \\
\text { ción profesional y de aptitud para el } \\
\text { empleo } \\
\text { Trabajo de voluntariado }\end{array}$ & $\begin{array}{l}\text {-Incremtar la autosuficiencia econó- } \\
\text { objetivos }\end{array}$ \\
& $\begin{array}{l}\text {-Aumentar las competencias y los com- } \\
\text { portamientos relacionados con el des- } \\
\text { empeño laboral } \\
\text {-Aumentar la sensación de contribu- } \\
\text { ción y de propósito }\end{array}$ \\
\hline
\end{tabular}

El asistente personal elige en la aplicación un dominio de CV y ve en su pantalla los deseos y metas de la persona con respecto a dicho dominio, así como las correspondientes necesidades de apoyos tal como se expresan en los ítems de la SIS, según el formato que figura en la Tabla 1. A continuación, puede redactar la instrucción sobre cómo apoyar a la persona en cada ítem de la SIS/necesidad de apoyo. Al redactar la instrucción en el PIA, se puede distinguir entre los apoyos para la persona con respecto a sus necesidades continuas de apoyo y los apoyos que tienen un objetivo claro en relación con la mejora del aprendizaje. En la aplicación, se puede elegir cualquiera de estas dos posibilidades. En el segundo caso, hay un formato para describir las instrucciones: se le pide al usuario de la aplicación que describa el objetivo, el qué, el cómo, el cuándo y el quién.

\section{Componente 4: puesta en práctica}

En esta fase, se pone en práctica el PIA por los profesionales encargados de prestar los apoyos a la persona. El PIA, tal como ha sido redactado por el asistente personal, es un compendio bastante completo de las necesidades de apoyo de la persona y de qué apoyos se le deben prestar por cada ítem.

Sin embargo, en función de la priorización realizada por la persona de sus deseos, ella y sus representantes legales ven en su pantalla (después de entrar con el nombre de usuario y la contraseña) una imagen un poco más elaborada pero, aun así, bastante compacta del PIA. Esta debería ser la información que es necesaria para prestar a la persona los apoyos de cada día.

Tanto la persona como los profesionales encargados de los apoyos pueden también hacer clic en la versión completa del PIA, lo cual puede ser necesario en situaciones que no es habitual encontrar todos los días.

Al dar los apoyos a la persona puede tenerse la sensación de que las instrucciones que figuran en el PIA no son las correctas o que es necesario ajustarlas. En tal caso, es posible comunicarlo al asistente personal para que pueda hacer los ajustes necesarios. 


\section{Componente 5: supervisión}

Es esencial supervisar continuamente el plan. La cuestión es si los apoyos que la persona está recibiendo son o no los adecuados: 1) ¿la forma en la que se está ayudando a la persona contribuye a alcanzar sus deseos, metas y aspiraciones personales? y 2) ¿son los apoyos que la persona está recibiendo una forma adecuada de responder a sus necesidades de apoyos? El hecho de que los deseos sean formulados de forma compacta tiene como objetivo que sean útiles para contribuir a este propósito. Y, en el caso de que haya apoyos que tengan un objetivo claro de mejorar el aprendizaje, el objetivo, el qué, el cómo, el cuándo y el quién se formulan de tal modo que sea posible realizar la evaluación sobre esta base. En el caso de los apoyos para la persona en relación con su necesidad continua de apoyos, la pregunta es clara: ¿son estos apoyos los adecuados?, ¿son la respuesta a las necesidades de apoyos que tiene la persona?

\section{Componente 6: evaluación del Plan Individual de Apoyos}

Cada año y medio, se miden los resultados de los apoyos con la Escala de Resultados Personales para hacerse una idea de los cambios en los resultados personales relacionados con la calidad de vida. Luego, estos resultados se comparten con el usuario y su asistente personal. Uno y otro pueden hablar sobre los resultados en el marco de su diálogo continuo, por lo que pueden utilizarse para hacer ajustes $\mathrm{y}$, por tanto, mejorar la calidad del PIA.

La Escala de Resultados Personales (Van Loon et al., 2008) se basa en el marco conceptual de la CV de Schalock y Verdugo (2002). La aplicación adecuada de la Escala de Resultados Personales (POS) posibilita la evaluación de los indicadores de CV relacionados con los ochos dominios centrales (y universales) de CV (Independencia: Desarrollo personal, Autodeterminación; Participación social: Relaciones interpersonales, Inclusión social; Derechos y Bienestar: Bienestar emocional, Bienestar físico, Bienestar material). Preferiblemente, esta evaluación debe hacerse mediante una entrevista con la persona (autoinforme), pero, si la persona no puede hablar por sí misma, la entrevista debe hacerse con un profesional o con uno de los padres (informe por un tercero). La POS tiene 6 ítems para cada dominio de CV. A efectos de comparación, el contenido de cada ítem es el mismo en las versiones de autoinforme y de informe por un tercero. En la versión 2.0 de la POS-A, nosotros añadimos un apartado titulado Comentarios Cualitativos. El entrevistador utiliza este apartado para registrar los comentarios y afirmaciones de la persona entrevistada con respecto a lo que es importante para su vida, qué metas personales tiene, qué es necesario mejorar en su calidad de vida y qué debe mejorarse en los apoyos que está recibiendo. Así, la escala da puntuaciones sobre tres factores y con ocho dominios de calidad de vida y una visión general de los comentarios cualitativos para cada dominio de CV. En la Figura 2 se presenta un ejemplo de una parte de los resultados de la POS para una persona concreta. 
FigURA 2. Ejemplo de parte de los resultados de la POS

\begin{tabular}{|lllll|}
\hline Factor & Domain & Question & Self & Other \\
\hline Independence & Self-Determination & 1 & 2 & 2 \\
& 2 & 3 & 2 \\
& 3 & 2 & 1 \\
& 4 & 2 & 3 \\
& 5 & 2 & 2 \\
Totals for this domain & 6 & 3 & 3 \\
\hline
\end{tabular}

\section{Remarks}

Self-Report: - My self determination is depending on the person who is supporting me. I do not like that.

- Advice me, but let me make my own decisions.

- I sometimes have difficulties to advocate for myself. A training might be good in this.

- The reaction of others can stop me giving my own opinion.

Report of Others: - Clear structure and boundaries seems to be important for Johnny.

\begin{tabular}{|c|c|c|c|}
\hline \multirow{7}{*}{ 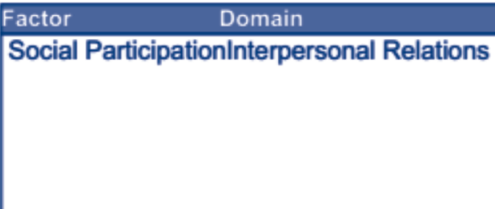 } & Question & Self & Other \\
\hline & 1 & 2 & 2 \\
\hline & 2 & 1 & 2 \\
\hline & 3 & 2 & 2 \\
\hline & 4 & 2 & 2 \\
\hline & 5 & 2 & 2 \\
\hline & 6 & 2 & 2 \\
\hline Totals for this domain & & 11 & 12 \\
\hline
\end{tabular}

\section{Remarks}

Self-Report: - I miss friends around.

Report of Others: - More contact with parents (besides telephone calls).

\begin{tabular}{|c|c|c|c|}
\hline $\begin{array}{l}\text { Factor } \quad \text { Domain } \\
\text { Social ParticipationSocial Inclusion }\end{array}$ & Question & Self & Other \\
\hline \multirow{6}{*}{ Social ParticipationSocial Inclusion } & 1 & 1 & 2 \\
\hline & 2 & 1 & 1 \\
\hline & 3 & 3 & 3 \\
\hline & 4 & 1 & 3 \\
\hline & 5 & 1 & 2 \\
\hline & 6 & 2 & 2 \\
\hline Totals for this domain & & 9 & 13 \\
\hline
\end{tabular}

\section{Remarks}

Self-Report: - I would prefer to live in Middelburg or Goes.

- I like to change sports, because the atmosphere at the football is not so nice.

Report of Others: - Expand social network. Create possibilities to integrate into society.

\begin{tabular}{|c|c|c|c|}
\hline \multirow{2}{*}{$\begin{array}{ll}\text { Factor } & \text { Domain } \\
\text { Social ParticipationRights }\end{array}$} & Question & Self & Other \\
\hline & 1 & 3 & 3 \\
\hline & 2 & 3 & 2 \\
\hline & 3 & 2 & 1 \\
\hline & 4 & 3 & 2 \\
\hline & 5 & 3 & 1 \\
\hline & 6 & 1 & 1 \\
\hline Totals for this domain & & 15 & 10 \\
\hline
\end{tabular}

\section{Remarks}

Self-Report: - Tell me, explain to me what my rights are.

Report of Others: - Find the balance between his self-determination, while retaining the right support. 


\section{Conclusiones}

El uso de resultados basados en la evidencia tiene múltiples propósitos en el programa de Arduin, el más importante de los cuales es evaluar los resultados de la planificación centrada en la persona realizada mediante un Plan Individual de Apoyos. Dado que las puntuaciones de cada usuario en la POS se anotan en una aplicación web, pueden describirse de varias formas. Los datos se suman para obtener información de los RBE relativa a la persona, el programa de la organización o la organización como un todo. Por ejemplo, los resultados de la POS en el Sistema de Información de Gestión de Arduin pueden, en cualquier momento, mostrarse en puntuaciones sin procesar o en percentiles para cada usuario, para cada programa o para la organización entera. Se pueden hacer comparaciones fácilmente entre dos puntos temporales determinados. Esta información está asegurada mediante contraseñas y solo puede acceder a ella el personal autorizado de la organización.

A partir de los perfiles de la POS, por ejemplo, el psicólogo que participa en los apoyos a una persona puede ver primero el perfil de CV (en puntuaciones sin procesar), comparar este perfil con el de otros usuarios de la organización (en percentiles) y ver los comentarios cualitativos. Su tarea consiste en aconsejar al usuario/asistente personal sobre los apoyos necesarios. Asimismo, el asistente personal puede obtener la información sobre los resultados. Su tarea consiste en hablar de estos resultados con la persona o su representante legal y utilizar esta información para mejorar el PIA. En un segundo ejemplo, un directivo de la organización puede ver las puntuaciones medias en CV para todos los usuarios de una determinada localidad (en puntuaciones sin procesar) y cuál es la puntuación promedio en CV para todos los usuarios de esa localidad comparado con el promedio de los otros usuarios de la organización (en percentiles). Estos datos son importantes para analizar y discutir y, por tanto, pueden utilizarse para las mejoras cualitativas, tal como determinar qué otras competencias son necesarias para los profesionales de esta localidad. En tercer lugar, se puede ver la puntuación promedio en CV de todos los usuarios de una localidad determinada (en puntuaciones sin procesar). Después de más rondas de entrevistas con la POS en una organización, se puede observar la progresión a lo largo del tiempo para cada dominio de CV, categoría de edad, nivel de los apoyos, etc. Estos datos son también importantes para analizar y discutir con el fin de desarrollar estrategias para la mejora de la calidad centradas sobre el cambio y la política de la organización, para desarrollar competencias adicionales que quizás sean necesarias para los profesionales en general, para tomar decisiones sobre qué medidas deben adoptarse con respecto a los usuarios $\mathrm{y}$ a los profesionales y determinar los predictores relevantes de los resultados personales obtenidos.

De este modo, la alineación entre los deseos y las metas, las necesidades de apoyo evaluadas mediante la SIS, los componentes del Plan Individual de Apoyos y los resultados obtenidos en calidad de vida medidos con la POS proporcionan una oportunidad excelente de prestar apoyos a las personas metódicamente para mejorar su calidad de vida. Además de mejorar el proceso de planificación individual, la medición de la CV sirve para crear las condiciones para generar un Sistema de Información de 
Gestión, en el cual la labor clave de la organización, el apoyo a las personas y la mejora de calidad de vida de la gente a la que se prestan apoyos, está en el centro. De esta forma, los resultados personales pueden utilizarse como guía del cambio y de la mejora de la organización.

\section{Referencias bibliográficas}

Brown, I. y Brown, R. I. (2003). Quality of life and disability: An approach for community practitioners. London: Jessica Kingsley Publishers.

Claes, C., Van Hove, G., van Loon, J., Vandevelde, S. y Schalock, R. L. (2009). Eight principles for assessing quality of life-related personal outcomes. Social Indicators Research, 98 (1), 61-72.

O'BRIEN, J. y O'BRIEN, C. L. (1989). What's worth working for? Leadership for better quality buman services. Syracuse, NY: Responsive Systems Associates.

Schalock, R. L. (2014). Aligning Quality of Life Domains to Potential Support Strategies and Anticipated Effects. Handout at a meeting in Arduin, March 2014.

Schalock, R. L., Brown, R. T., Cummins, R., Mattikka, L., Felce, D. y Brown, I. (2000). Conceptualization, measurement and application of the concept of quality of life: A consensus document symposium. Journal of Intellectual Disability Research, 44, 452-452.

Schalock, R. L. y Verdugo, M. Á. (2002). Handbook on quality of life for human service practitioners. Washington, DC: American Association on Mental Retardation.

Schalock, R. L. y Verdugo, M. Á. (2012 a). A conceptual and measurement framework to guide policy development and systems change. Journal of Policy and Practice in Intellectual Disabilities, 7, 71-81.

Schalock, R. L., Verdugo, M. Á., Bonham, G. S., Fantova, F. y van Loon, J. (2008). Enhancing personal outcomes: Organizational strategies, guidelines, and examples. Journal of Policy and Practice in Intellectual Disabilities, 5 (4), 276-285.

Schalock, R. L., Verdugo M. Á. y Gómez, L. E. (2011). Evidence-based practices in the field of Intellectual and developmental disabilities: An international consensus approach. Evaluation and Program Planning, 34, 273-282.

Schalock, R. L., Verdugo, M. Á., Jenaro, C., Wang, M., Wehmeyer, M., Xu, J. y LaChaPELLE, Y. (2005). Cross-cultural study of quality of life indicators. American Journal on Mental Retardation, 110, 298-311.

Thompson, J. T., Bryant, B. R., Campbell, E. M., Craig, E. M., Hughes, C. M., Rotholz, D. A. et al. (2004). Support Intensity Scale Manual. Washington, DC: American Association on Mental Retardation.

Van Loon, J. H. M., Bonham, G. S., Peterson, D. D., Schalock, R. L., Claes, C. y DecraMER, A. E. M. (2012). The Use of Evidence-Based Outcomes in Systems and Organizations Providing Services and Supports to Persons with Intellectual Disability. Evaluation and Program Planning, 36 (2013), 80-87.

Van Loon, J. H. M., Claes, C., Van Hove, G. y Schalock, R. L. (2010). De ontwikkeling van de Persoonsgerichte Ondersteuningsuitkomsten Schaal (POS). Nederlands Tijdschrift voor de Zorg aan mensen met verstandelijke beperkingen, 36 (3), 180-196.

VAn LoOn, J. H. M. y VAN Hove, G. (2001). Emancipation and self-determination of people with learning disabilities and down-sizing institutional care. Disability E Society, 16, 233-254. 
Van Loon, J. H. M., Van Hove, G. y Schalock, R. L. (2009). The use of SIS data / items for the development of an individual supports plan. En R. L. Schalock y J. Thompson, The SIS Implementation Guide. Washington, DC: American Association on Intellectual and Developmental Disabilities.

Van Loon, J. H. M., Van Hove, G., Schalock, R. L. y Claes, C. (2008). Personal Outcomes Scale: A Scale to Assess an Individual's Quality of Life. Stichting Arduin, Netherlands: Universiteit Gent.

Vreeke, G. J., Janssen, C. G. C., Resnick, S. y Stolk, J. (1998). Kwaliteit van bestaan van mensen met een verstandelijke handicap. Ontwikkeling van een meetinstrument voor woonvoorzieningen en de stand van zaken in Nederlandse instellingen. Amsterdam: Vrije Universiteit.

Wang, M., Schalock, R. L., Verdugo, M. Á. y Jenaro, C. (2010). Examining the factor structure and hierarchical nature of the quality of life construct. American Journal on Intellectual and Developmental Disabilities, 115, 218-233. 